\title{
MENSURAÇÃO DA QUALIDADE DE SERVIÇO EM ACADEMIAS DE GINÁSTICA: DAS ESCALAS PADRONIZADAS AO DESENVOLVIMENTO DE UMA ESCALA ESPECÍFICA
}

\author{
E. Pereira Filho ${ }^{1}$, D. F. Campos ${ }^{2}$ e M. L. R. Dantas ${ }^{3}$ \\ ${ }^{1}$ Instituto Federal de Educação, Ciência e Tecnologia do RN (IFRN) \\ ${ }^{2,3}$ Universidade Potiguar (UnP) \\ E-mail: evadio.filho@ifrn.edu.br ${ }^{1}$
}

Artigo submetido em maio/2013 e aceito em outubro/2013

\section{RESUMO}

O presente estudo tem como objetivos: a) propor uma escala para mensurar a qualidade dos serviços ofertados por academias de ginástica; b) avaliar a dimensionalidade do construto qualidade do serviço; e c) identificar, com base na matriz importânciadesempenho, em quais dimensões os gestores devem focar sua atenção. Obedecendo a critérios de similaridade, completude, justaposição, aglutinação, especificidade e presença em trabalhos empíricos, 31 atributos foram selecionados para compor a escala proposta. Posteriormente, os atributos que integravam a escala foram submetidos ao crivo de especialistas e clientes. Estes analisaram os níveis de clareza e representatividade das sentenças definidoras de cada um dos atributos. Ao final deste processo, um teste piloto com 30 clientes foi desenvolvido, com intuito de realizar os últimos ajustes. Foram entrevistados 986 clientes de seis dentre as maiores academias do mercado de Natal/RN. Os resultados revelaram que a escala proposta, intitulada QSAG, apresentou uma consistência interna significativa (alfa de Cronbach foi $0,940)$. Da análise fatorial, seis fatores emergiram os quais explicam $61,82 \%$ da variância dos dados. Isto ratifica a multidimensionalidade do construto qualidade dos serviços. A utilização da matriz importânciadesempenho permitiu extrair um conjunto de ações que podem ser usadas para dar suporte às decisões gerenciais de aperfeiçoamento das estratégias de qualidade.

PALAVRAS-CHAVE: qualidade dos serviços, academias de ginástica, matriz importância-desempenho.

\section{MEASUREMENT OF SERVICE QUALITY IN HEALTH CLUBS: OF STANDARD SCALES TO DEVELOPMENT OF A SPECIFIC SCALE}

\begin{abstract}
The present study aims to: a) propose a scale to measure the service quality offered by health clubs; b) evaluate the dimensionality of the service quality construct; and c) identify, based on importanceperformance matrix, in which dimensions managers should focus their attention. Obeying similarity criteria, completeness, juxtaposition, agglutination, specificity and presence in empirical studies, 31 attributes were selected for compose the scale proposed. Subsequently, the attributes that formed the scale were analyzed by specialists and customers. These analyzed the levels of clarity and representativeness of sentences defining each attribute. Finalized this process, a pilot test with 30
\end{abstract}

customers was developed, in order to make the last adjustments. We interviewed 986 customers of six among the major health clubs of Natal/RN. The results revealed that the proposed scale, titled QSAG, showed a significant internal consistency (Cronbach's alpha was 0.940). The factor analysis, six factors emerged which explain $61.82 \%$ of data variance. This confirms the multidimensionality of the service quality construct. The use of importance-performance matrix allowed to extract a set of actions that can be used to support management decisions for improvement of quality strategies.

KEYWORDS: service quality, health clubs, importance-performance matrix. 


\section{MENSURAÇÃO DA QUALIDADE DE SERVIÇO EM ACADEMIAS DE GINÁSTICA: DAS ESCALAS PADRONIZADAS AO DESENVOLVIMENTO DE UMA ESCALA ESPECÍFICA}

\section{INTRODUÇÃO}

O atual estilo de vida caracterizado pelo sedentarismo, altos níveis de estresse, nutrição desbalanceada e vícios, como o álcool e o tabagismo, vem despertando nas pessoas uma preocupação com as condições do seu corpo, principalmente, no tocante à saúde e à estética. Dentre as medidas adotadas por elas para tornarem a sua rotina mais salutar, a aderência à prática de atividades físicas sobressai (AGUIAR, 2007; PEDRAGOSA, CORREIA, 2009; YILDIZ, 2011; YILDIZ, KARA, 2012). As academias de ginástica, então, surgiram como opção para a população urbana praticar exercícios físicos (UHLMANN et al., 2009; OSTI, 2009). Neste contexto, é nítida a tendência de crescimento da indústria do fitness ${ }^{1}$ a qual possui como atores não só academias de ginástica, como também empresas especializadas em vestuário, acessórios e equipamentos, segmentadas de acordo com a modalidade seguida pelo praticante (AFTHINOS, THEODORAKIS, NASSIS, 2005; BERTEVELLO, 2006; PINHEIRO, PINHEIRO, 2006; LAGROSEN, LAGROSEN, 2007; FURTADO, 2009; YILDIZ, 2011; SOITA, 2012).

Em 2011, no âmbito mundial, o fitness gerou aproximadamente 72,7 bilhões de dólares em receita, em mais de 133.000 empreendimentos que prestaram serviços a 129 milhões de membros (IHRSA, 2012). O Brasil, com 18.000 estabelecimentos e faturamento próximo a R\$ 2 bilhões por ano, ocupa o segundo lugar em número de academias, atrás somente dos EUA (ACAD, 2011). Em Natal/RN, dados do Conselho Regional de Educação Física mostram 196 empresas registradas (CREF, 2012). Os números revelam um mercado atrativo e cada vez mais competitivo. Entretanto, à medida que cresce a oferta, os usuários das academias de ginástica tornam-se mais exigentes em relação aos serviços ofertados. É comum, ademais, neste segmento, o gestor enfrentar demandas sazonais, rotatividade dos usuários entre as academias e o abandono da prática de exercícios físicos (TRIADO, APARÍCIO, RIMBAU, 1999; AGUIAR, 2007; OSTI, 2009).

As organizações necessitam investir cada vez mais em qualidade de serviço para manter seus clientes satisfeitos. Para Barreto et al. (2010), não basta apenas captar os clientes e oferecer-Ihes o básico, mas sim estudar o seu comportamento e encantá-los. Logo, este artigo tem como objetivos: a) propor uma escala para mensurar a qualidade dos serviços ofertados por academias de ginástica; b) avaliar a dimensionalidade do construto qualidade do serviço e c) apontar, com base na matriz importância-desempenho, em quais dimensões os gestores devem focar sua atenção, com o propósito de aperfeiçoar as estratégias de qualidade.

\section{FUNDAMENTAÇÃO TEÓRICA}

Na literatura, a corrente de pensamento que defende o paradigma da desconfirmação entende que a qualidade do serviço é a medida de quão bem o nível do serviço prestado atende às expectativas do consumidor. Nesta linha, Grönroos (1984) desenvolveu um modelo bidimensional, enaltecendo a qualidade técnica (aquilo que o cliente recebe como resultado do

\footnotetext{
${ }^{1}$ Para Saba (2006), o termo fitness está relacionado ao condicionamento físico e atlético do indivíduo. Tem como principal objetivo o desempenho estético. É impulsionado por aqueles que anseiam os padrões de beleza socialmente valorizados.
} 
processo de produção) e a qualidade funcional (a forma como o consumidor recebe o resultado técnico do serviço). Para o autor, a qualidade do serviço consiste num processo comparativo entre o serviço esperado (expectativas) e o serviço experimentado (percepções de desempenho). Este discernimento coaduna com as proposições de Parasuraman, Zeithaml e Berry (1985), ao formatarem o modelo das lacunas de qualidade. Este modelo estabelece cinco lacunas (gaps) de qualidade sob as diferentes percepções dos autores envolvidos no processo (gestores, operadores e clientes). A lacuna 5, diferença entre as expectativas do cliente e o serviço por ele percebido, seria a resultante das demais. Destarte, discrepâncias ocorridas nas demais lacunas refletirão numa desconformidade entre o serviço esperado e o desempenho percebido pelo consumidor, acarretando insatisfação (BOWERSOX, CLOSS, COOPER, 2006; PARASURAMAN, ZEITHAML, BERRY, 1985). Grönroos (1984) ressalta que os gestores devem compreender a qualidade do mesmo modo que os consumidores o fazem, evitando, assim, tomar decisões equivocadas e desperdiçar tempo e dinheiro.

\section{ESCALAS DE MENSURAÇÃO DA QUALIDADE DOS SERVIÇOS}

Em 1985, Berry, Parasuraman e Zeithaml relataram que a qualidade do serviço era um fenômeno multidimensional e identificaram dez determinantes utilizados pelos consumidores para sua avaliação. Este quantitativo foi reduzido para cinco (tangibilidade, confiabilidade, presteza, segurança e empatia), em meados de 1988, momento em que sugeriram a escala SERVQUAL. Esta escala representa a construção de uma abordagem padronizada voltada para medir as percepções dos usuários em relação à qualidade do serviço, tendo como base a discrepância entre expectativas e desempenho (PARASURAMAN, ZEITHAML, BERRY, 1985; CRONIN, TAYLOR, 1992; GRÖNROOS, 2009).

Não obstante a sua relevância para o desenvolvimento de pesquisas na área de qualidade dos serviços, o instrumento SERVQUAL tem sido objeto de críticas, conceituais e operacionais, conforme relatado por Akbaba (2006). Dentre as objeções, a generalização da estrutura das cinco dimensões sobressai. Grönroos (2009) afirma que alguns estudos revelaram que as cinco dimensões do SERVQUAL são universais para as diversas tipologias de serviço, enquanto outros não sustentaram este conjunto padrão. Yildiz e Kara (2012) expõem que o referido instrumento é contestado pelas inconsistências na dimensionalidade.

Para Akbaba (2006) e Colmenares e Saavedra (2007), as dimensões e atributos, que integram o SERVQUAL, necessitam ser contextualizados. Faz-se necessário, portanto, customizálos para o setor em que a investigação será procedida. Por estas razões, Babakus e Boller (1992) mencionaram que não é frutífero prosseguir no desenvolvimento de uma escala padrão de medição de qualidade, e que seja aplicável a uma ampla variedade de serviços.

Em virtude da imprecisão do conceito de expectativas, alguns teóricos, como Cronin e Taylor (1992) e Babakus e Boller (1992), salientaram a necessidade de desenvolver uma escala, metodologicamente, mais precisa. A escala SERVPERF surgiu para preencher esta fenda. Desenvolvida e testada inicialmente por Cronin e Taylor (1992), é uma ferramenta alternativa para mensurar a qualidade dos serviços numa escala atitudinal, a partir unicamente da medida do desempenho do serviço. Logo, o SERVPERF, por ser uma variante da escala SERVQUAL, contempla os 22 itens que formam a subescala "desempenho" (CRONIN, TAYLOR, 1992; JAIN, GUPTA, 2004). 
Para Cronin e Taylor (1992), a escala SERVPERF é mais eficiente do que a SERVQUAL, uma vez que reduz em $50 \%$ o quantitativo de itens que devem ser medidos, passando de 44 para 22 afirmações. Isto ameniza os efeitos de enfado que o tamanho do questionário pode proporcionar ao ser aplicado. No entanto, Parasuraman, Zeithaml e Berry (1994) apresentaram críticas à escala SERVPERF, ao reafirmarem que a qualidade do serviço está centrada na discrepância entre expectativas e percepções de desempenho. Para estes, medidas baseadas no paradigma da desconfirmação fornecem informações mais ricas e têm maior valor de diagnóstico para os gestores.

Face ao exposto, percebe-se que não há um consenso, na literatura, sobre qual das escalas, SERVPERF ou SERVQUAL, é a mais eficaz e apropriada (BRADY, CRONIN, BRAND, 2002; JAIN, GUPTA, 2004). Nota-se, apenas, que é crescente a preocupação dos gestores com a variável qualidade, conforme apontam Andronikidis e Bellou (2010) ao afirmarem que o papel da qualidade do serviço para a sobrevivência e êxito da empresa é de extrema importância. Logo, as escalas apresentadas nesta subseção representam um dos meios que permitem aos gestores diagnosticar quais são as principais deficiências de qualidade e, consequentemente, traçar ações mitigadoras.

\section{ESTUDOS EMPÍRICOS}

Não obstante a intangibilidade que caracteriza os serviços, a sua especificação pode ser alcançada através de atributos operacionalizáveis e mensuráveis. Para Yildiz (2011), as dimensões e os atributos dos serviços variam de segmento a segmento, por isso fazer uso de um instrumento universal de medição da qualidade do serviço como, por exemplo, o SERVQUAL, mostra-se inadequado, pois este não retrata características peculiares ao setor estudado.

Diversos trabalhos na área de fitness apresentaram atributos e escalas específicas voltadas para medir a qualidade dos serviços. Chelladurai, Scott e Haywood-Farmer (1987) desenvolveram uma escala, comumente conhecida como SAFS (Scale of Attributes of Fitness Services), com intuito de medir o grau de influência de determinados fatores na decisão de compra dos serviços de academias de ginástica. Ao coletar dados junto a 436 clientes de academias situadas no Canadá, observou-se que o instrumento apresentou uma consistência interna significativa, além de contemplar cinco dimensões.

Por sua vez, Kim e Kim (1995) elaboraram um instrumento, intitulado de QUESC (Quality Excellence of Sports Centers), para avaliar a qualidade dos serviços prestados em centros esportivos. Este consistiu em uma escala de 45 itens, listados em dois questionários distintos, em que o primeiro apreendia as expectativas dos clientes, e o segundo avaliava o nível de serviço prestado pelo centro esportivo na percepção dos respondentes. A pesquisa envolveu 271 membros de centros esportivos situados na Coréia, e apontou que as doze dimensões se mostram confiáveis, assim como a escala foi percebida como válida.

Triado, Aparicio e Rimbau (1999) realizaram um estudo com base em uma ferramenta de sua autoria e constituída por treze variáveis. Avaliaram uma amostra composta por 698 clientes de centros esportivos situados em Barcelona, na Espanha. Como resultado, cinco dimensões emergiram da análise fatorial, sendo elas: qualidade das instalações, qualidade dos recursos humanos, custo, comunicação e a importância do ambiente social. Já Alexandris e Palialia (1999) realizaram um estudo com 210 clientes de academias de ginástica localizadas na Grécia, com intuito de avaliar a satisfação do cliente. Para tal, desenvolveram uma escala, com 20 itens e 
cinco dimensões (instalações/programas, individual/psicológico, relaxamento, social e saúde/fitness). O construto apresentou uma consistência interna significativa, bem como sustentou a multidimensionalidade da satisfação.

Lam, Zhang e Jensen (2005), por sua vez, desenvolveram uma escala conhecida como SQAS (Service Quality Assessment Scale). Foi aplicada junto a 1202 membros de academias de ginástica situadas nos Estados Unidos. Como principal resultado, ficou constatada a multidimensionalidade da qualidade, uma vez que seis dimensões emergiram da análise fatorial. Yildiz (2011) examinou a percepção dos clientes sobre a qualidade dos serviços prestados pelas academias de ginástica na Turquia, empregando, conjuntamente, a escala SQS-FC (Service Quality Scale for Fitness Centers), de sua autoria, e a matriz importância-desempenho. Participaram da pesquisa 246 frequentadores de uma academia privada. Identificou um significativo déficit de qualidade percebida na dimensão ambiente físico, demandando uma ação urgente. Isto se justifica em razão do desempenho percebido pelos clientes estar aquém de suas expectativas, bem como por esta dimensão integrar o grupo dos atributos mais importantes. Destarte, existia a necessidade de aperfeiçoar, prioritariamente, os aspectos físicos das academias de ginástica por meio da realocação de recursos.

A Figura 1 apresenta um resumo dos 23 estudos empíricos, nacionais e internacionais, que serviram como base para a identificação dos principais atributos e dimensões relacionados aos serviços de academia de ginástica.

\begin{tabular}{|c|c|c|c|c|c|}
\hline Autor/Ano & País & A* & Autor/Ano & País & A $^{* 1}$ \\
\hline $\begin{array}{c}\text { Chelladurai, Scott e Haywood- } \\
\text { Farmer (1987) }\end{array}$ & Canadá & 436 & Aguiar (2007) & Brasil & 347 \\
\hline Kim, Kim (1995) & Coréia & 271 & Uhlmann et al. (2009) & Brasil & 105 \\
\hline Alexandris, Palialia (1999) & Grécia & 210 & Teixeira, Correia (2009) & Portugal & 130 \\
\hline Triado, Aparicio, Rimbau (1999) & Espanha & 698 & Pedragosa, Correia (2009) & Portugal & 463 \\
\hline $\begin{array}{c}\text { Papadimitrious, Karteroliotis } \\
\text { (2000) }\end{array}$ & Grécia & 487 & Osti (2009) & Brasil & 318 \\
\hline $\begin{array}{c}\text { Murray, Howat (2002) } \\
\text { Theodorakis et al. (2004) }\end{array}$ & Austrália & 218 & Barreto et al. (2010) & Brasil & 92 \\
\hline $\begin{array}{c}\text { Afthinos, Theodorakis, Nassis } \\
\text { (2005) }\end{array}$ & Grécia & 346 & Yildiz (2011) & Turquia & 246 \\
\hline Lam, Zhang, Jensen (2005) & EUA & 1202 & Moreno, García, Pomar (2012) & Espanha & 261 \\
\hline Dias (2006) & Portugal & 108 & Yildiz, kara (2012) & Turquia & 164 \\
\hline Bodet (2006) & França & 184 & Soita (2012) & Uganda & 602 \\
\hline Lagrosen, Lagrosen (2007) & Suécia & 15 & \multicolumn{2}{|c|}{ Legenda: A* = tamanho da amostra. } \\
\hline
\end{tabular}

Figura 1 - Estudos empíricos que permitiram elencar atributos e dimensões do serviço de academias de ginástica. Fonte - Elaborada pelos Autores.

\section{METODOLOGIA}

O trabalho de pesquisa foi balizado pela literatura sobre gestão de serviços e estudos empíricos sobre a qualidade dos serviços em academias de ginástica. O esquema metodológico adotado no desenvolvimento da pesquisa é apresentado na Figura 2. 


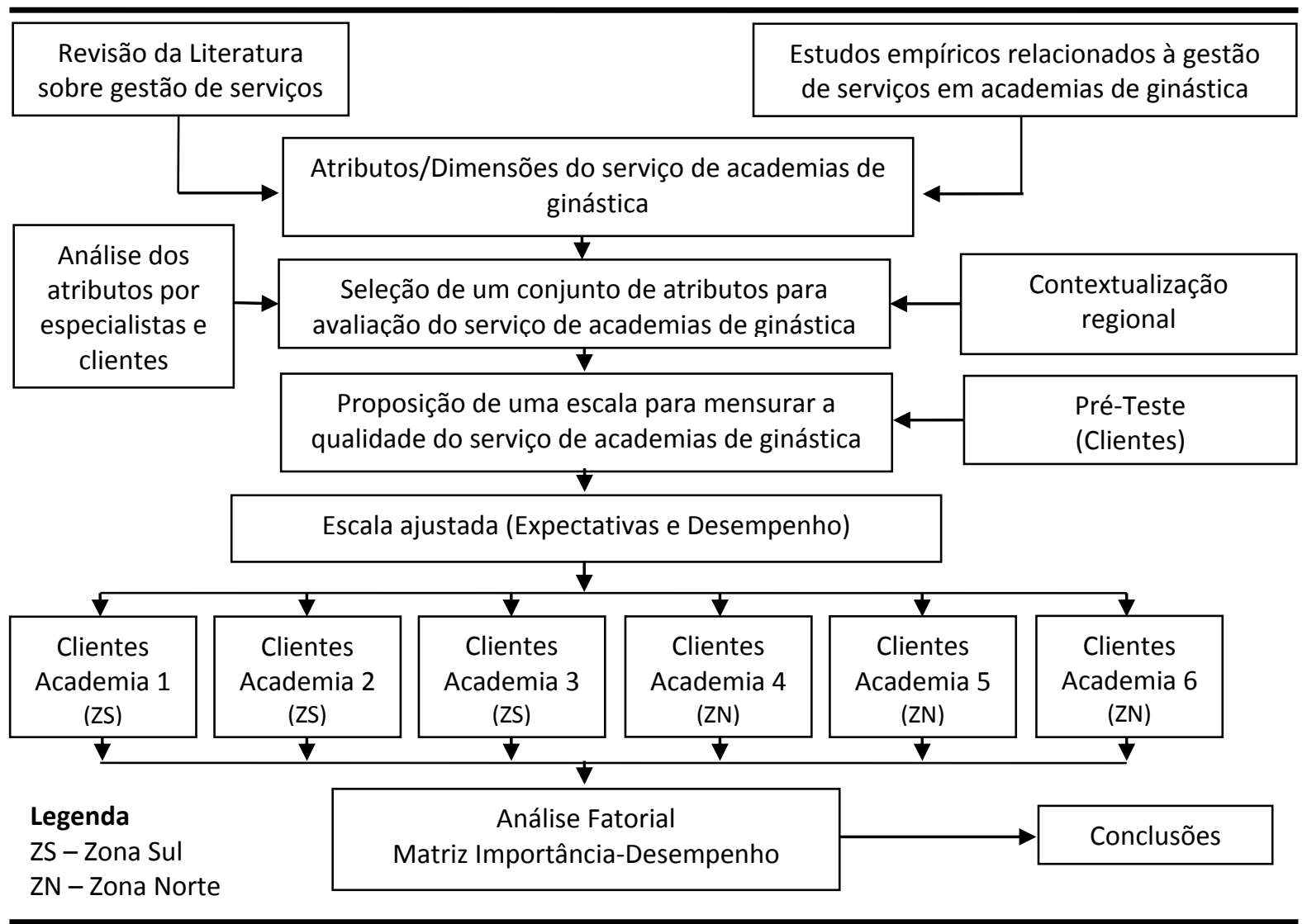

Figura 2 - Esquema metodológico.

Fonte - Elaborada pelos Autores.

Com base no referencial teórico abordado, foram coletados, livremente, 643 atributos. Apoiado no critério de similaridade, as repetições foram eliminadas, de modo que o número de atributos foi reduzido para 176. Uma segunda análise, com base nos conteúdos das sentenças definidoras, foi empreendida com o objetivo de reduzir o conjunto de atributos. Para tanto, utilizou-se os critérios de completude, justaposição, aglutinação e especificidade. Por exemplo, um atributo estrutura da academia é demasiadamente genérico. $O$ atributo altura das salas (pé direito) é demasiadamente específico. Estrutura da academia pode ser entendida como estacionamento, acessos, prédios, equipamentos ou até capacidade de atendimento. Por sua vez, o atributo equipamentos pode envolver quantidade, qualidade, variedade, estado de conservação, programa de manutenção. A análise de conteúdo do conjunto de atributos coletados na literatura é vital para a consolidação de um conjunto que abarque as diferentes medidas da qualidade e viabilize o instrumento e o processo de coleta de dados.

\section{ATRIBUTOS UTILIZADOS}

Um grupo, constituído por 17 clientes e 42 especialistas na área, analisou os 31 atributos selecionados, com base nos níveis de compreensão/clareza e representatividade de cada um deles. Neste processo de validação, os respondentes também puderam propor a substituição/inserção/supressão de algum termo utilizado nas sentenças definidoras dos atributos a fim de adequar a linguagem ao contexto local. Ao final, foram requisitados, ainda, que os especialistas e clientes apontassem outros atributos não contemplados na relação inicial e 
considerados relevantes para a avaliação da qualidade dos serviços de academias de ginástica. Face às sugestões ofertadas, foram reescritos nove atributos (horários das modalidades, atendimento dos instrutores, atendimento dos funcionários administrativos, variedade das modalidades, interesse, quantidade de equipamentos, variedade e modernidade dos equipamentos, programa de treinamento e avaliações). A Figura 3 aponta os 31 atributos contidos na escala proposta e já com as devidas ponderações feitas pelos especialistas e clientes.

\section{ATRIBUTOS (SIGLA)}

Conservação: Estado de conservação dos espaços físicos, mobílias e equipamentos da academia. (Q1)

Tamanho e Arquitetura dos Ambientes: Espaços e outros elementos arquitetônicos dos ambientes da academia (recepção, salas, vestiários, banheiros, entre outros). (Q2)

Estacionamento: Disponibilidade e segurança do estacionamento. (Q3)

Vestiários e Banheiros: Quantidade de vestiários e banheiros disponíveis na academia. (Q4)

Variedade e Modernidade dos Equipamentos: Diversidade e modernidade de máquinas de musculação, aparelhos para treinamento aeróbico, pesos livres e demais acessórios. (Q5)

Quantidade de Equipamentos: Quantidade de máquinas de musculação, aparelhos para treinamento aeróbico, pesos livres e demais acessórios. (Q6)

Arranjo físico: Disposição das mobílias e equipamentos nos ambientes da academia. (Q7)

Horários das Modalidades: Horários e tempo de duração das atividades oferecidas (musculação, ginástica, spinning, artes marciais, atividades aquáticas, entre outras). (Q8)

Variedade das Modalidades: Variedade de atividades oferecidas (musculação, ginástica, spinning, artes marciais, atividades aquáticas, entre outras). (Q9)

Serviços Acessórios: Serviços acessórios ofertados na academia, tais como lanchonete, loja de artigos esportivos, orientação nutricional, personal trainer, entre outros. (Q10)

Avaliações: Avaliação física ofertada pela academia. (Q11)

Localização: Localização da academia. (Q12)

Limpeza: Limpeza e higienização dos ambientes e equipamentos da academia. (Q13)

Ventilação: Conforto térmico nos ambientes da academia (salas, vestiários, banheiros, entre outros). (Q14)

Iluminação: lluminação dos ambientes da academia (salas, vestiários, banheiros entre outros). (Q15)

Música: Agradabilidade da música ambiente e adequação do volume. (Q16)

Acesso: Facilidade de acesso à academia, desde o estacionamento até seus espaços internos, maquinários e mobílias (bebedouros, televisores, entre outros). (Q17)

Segurança: Condições de segurança aos clientes (presença de vigilantes, circuito interno de TV, entre outros). (Q18)

Programa de Treinamento: Prescrição de exercícios físicos pelo instrutor de acordo com os objetivos do cliente. (Q19)

Atendimento dos Instrutores: Atendimento dos instrutores da academia (cortesia, presteza, atenção e simpatia). (Q20)

Qualificação: Conhecimento técnico e prática profissional dos instrutores para executar suas atividades e entender as necessidades, limitações e potencialidades dos clientes. (Q21)

Instruções: Clareza das informações ofertadas pelos instrutores aos clientes (instruções de como usar os equipamentos, a forma correta de executar o exercício, entre outras). (Q22)

Marca: Imagem e reputação da academia no mercado. (Q31)

Preço: Valor pago pelo cliente referente aos serviços prestados pela academia (custo $x$ benefício). (Q29)

Interesse: $\mathrm{O}$ interesse dos instrutores no progresso dos clientes, aconselhando-os em relação aos programas de treino e uso de equipamentos, além de fornecer informações sobre o seu desenvolvimento. (Q23)

Quantitativo de Funcionários: Quantidade de instrutores, recepcionistas e pessoal de apoio para atender a demanda. (Q24)

Atendimento dos Funcionários Administrativos: Atendimento dos recepcionistas e pessoal de apoio da academia (cortesia, presteza, atenção e simpatia). (Q25)

Reclamações: Forma como os funcionários recebem, registram, tratam e dão resposta às reclamações apresentadas pelos clientes. (Q26)

Aparência: Aparência dos funcionários (limpa, arrumada e profissional). (Q27)

Emergência: Capacidade da equipe em lidar com situações de emergência (procedimentos de primeiros 
socorros em casos de machucados, cortes, fraturas, quedas, desmaios, infartos, entre outros). (Q28)

Convênios, Ações de Fidelização e Planos: Convênios firmados, programas/ações de fidelização e planos

ofertados pela academia. (Q30)

Figura 3 - Lista dos atributos que fazem parte da escala proposta.

Fonte - Elaborada pelos Autores.

\section{INSTRUMENTO DE COLETA}

O instrumento de coleta foi composto por três módulos. O primeiro contemplou questões voltadas aos aspectos sociodemográficos e motivacionais dos respondentes. O segundo módulo foi planejado para apreender o nível de expectativas dos respondentes, mediante a atribuição do grau de importância para cada um dos 31 atributos incluídos na pesquisa. No terceiro módulo, o respondente apontou a sua percepção sobre o serviço recebido na academia ao qual estava vinculado, em relação aos mesmos atributos incluídos no módulo 2. A percepção do serviço recebido corresponde ao desempenho da academia avaliada. Nos últimos dois módulos, as respostas foram obtidas mediante a utilização de uma escala Likert de 11 pontos, variando de zero (não importante/péssimo desempenho) a dez (muito importante/excelente desempenho). Um teste piloto foi realizado com 30 clientes, sendo cinco de cada uma das academias pesquisadas. Este procedimento permitiu realizar os últimos ajustes no instrumento de coleta.

\section{UNIVERSO E AMOSTRA}

O universo de pesquisa foi constituído pelos 2.180 clientes matriculados em seis academias de ginástica, dentre as maiores com atuação nas zonas norte e sul de Natal/RN, de acordo com os registros no Conselho Regional de Educação Física (10ạ Região). Estas zonas administrativas foram escolhidas por apresentarem o maior número de academias de ginástica em funcionamento. A seleção das organizações obedeceu a critérios geográficos, de conveniência e acessibilidade. As amostras foram calculadas por academia, de forma independente, considerando o número de matriculados em cada uma delas. O cálculo amostral foi desenvolvido admitindo-se um erro de 6\% e um nível de confiança de 95\%, conforme ilustra a Tabela 1.

Tabela 1 - Universo e amostra.

\begin{tabular}{|c|c|c|c|c|}
\hline Empresas (Sigla) & Zona & Universo & Amostra* & Quantidade planejada \\
\hline Academia 1 (A1) & Sul & 500 & 175 & 190 \\
\hline Academia 2 (A2) & Sul & 500 & 175 & 190 \\
\hline Academia 3 (A3) & Sul & 250 & 130 & 145 \\
\hline Academia 4 (A4) & Norte & 350 & 152 & 167 \\
\hline Academia 5 (A5) & Norte & 260 & 132 & 147 \\
\hline Academia 6 (A6) & Norte & 320 & 146 & 161 \\
\hline \multicolumn{2}{|l|}{ Total } & 2.180 & 910 & 1.000 \\
\hline
\end{tabular}

Os respondentes foram selecionados por critérios de conveniência e acessibilidade. Dos 1.000 questionários aplicados, apenas 986 foram considerados válidos por apresentarem todas as questões marcadas, com indicação de uma única resposta. 


\section{COLETA E TRATAMENTO DOS DADOS}

Os clientes foram entrevistados no mês de dezembro de 2012, por uma equipe composta por seis entrevistadores devidamente treinados para realizar a coleta. A equipe foi igualmente distribuída entre as academias participantes, sendo cada um dos entrevistadores responsável pela coleta dos dados da academia a qual foi destinado. Foram instruídos a abordarem os clientes antes ou depois do treino, e realizar a coleta nos horários de maior fluxo: das $06 \mathrm{~h}$ às $10 \mathrm{~h}$ e das $15 \mathrm{~h}$ às $22 \mathrm{~h}$.

Uma análise fatorial foi desenvolvida para avaliar a dimensionalidade do construto qualidade do serviço. A matriz importância-desempenho também foi empregada, visando identificar em quais dos fatores extraídos (dimensões) ocorreram déficits de qualidade. Isto permite orientar os gestores sobre quais diretrizes seguir para melhorar a prestação do serviço.

\section{APRESENTAÇÃO E DISCUSSÃO DOS RESULTADOS}

Os resultados aqui apresentados refletem a apuração de 986 respostas válidas de 1000 questionários aplicados. O tratamento estatístico foi desenvolvido utilizando-se o Statistical Package for the Social Science - SPSS, versão 19.0. Para alcançar os objetivos propostos, foram utilizadas a análise fatorial para identificar as dimensões da qualidade do serviço e o alfa de Cronbach que indica o nível de consistência interna da escala proposta. Sequencialmente, foi empregada a matriz importância-desempenho com intuito de identificar em quais dimensões da qualidade os gestores devem concentrar esforços para aprimorar os serviços ofertados.

A escala proposta, formada por 31 variáveis, apresentou um Alfa de Cronbach de 0,940. Este valor, segundo Hair Junior et al (2009), é aceitável e indica a alta confiabilidade do construto. Consequentemente, como a escala se mostrou um instrumento de medição adequado, foi nomeada como QSAG (Escala para mensurar a qualidade dos serviços ofertados por academias de ginástica).

A análise fatorial foi aplicada com os valores que expressam os gaps de qualidade (notas de desempenho de cada um dos respondentes subtraídas das respectivas notas de importância), utilizando-se o método de extração dos componentes principais e rotação Varimax. Conforme a Tabela 2, os valores obtidos nos testes $\operatorname{KMO}(0,926)$ e de esfericidade de Bartlett $(6200,619$, significativo a 0,000 ) mostram a adequação da amostra à técnica. Quanto às comunalidades de cada uma das variáveis, os índices oscilaram entre 0,388 e 0,767 , o que mostra que as variáveis explicam adequadamente o fator. Estes valores estão em conformidade com os padrões sugeridos por Hair Junior et al (2009).

Tabela 2 - Testes KMO e Bartlett.

\begin{tabular}{c|cc}
\hline Medida de adequação de Kaiser-Meyer-Olkin (KMO) & \multicolumn{2}{|c}{0,926} \\
\hline & Aprox. Qui Quadrado & 6200,619 \\
Teste de esfericidade de Bartlett & $\begin{array}{c}\text { Graus de liberdade (gl) } \\
\text { Significância }\end{array}$ & 465 \\
& \multicolumn{2}{c}{ Fonte - Pesquisa (2012). }
\end{tabular}

A partir da análise fatorial, as 31 variáveis foram agrupadas em seis fatores que explicam $61,82 \%$ da variância dos dados originais (Tabela 3). Nove itens foram carregados na dimensão 1 (D1), a qual foi nomeada estrutura física e conveniência. Seis foram carregados na dimensão 2 (D2, intitulada instrutores e programa de treinamento), sete na dimensão 3 (D3, batizada 
conforto e equipamentos), seis na dimensão 4 (D4, nomeada preço, reclamações e emergência), dois na dimensão 5 (D5, intitulada funcionários administrativos e aparência) e um na dimensão 6 (D6, nomeada localização). Estes dados reforçam a multidimensionalidade do construto qualidade do serviço, abordada, dentre outros autores, por Parasuraman, Zeithaml e Berry (1985), Chelladurai, Scott e Haywood-Farmer (1987) e Kim e Kim (1995).

Tabela 3 - Dimensões extraídas da análise fatorial.

\begin{tabular}{|c|c|c|c|c|c|c|}
\hline Atributos da escala & D1 & D2 & D3 & D4 & D5 & D6 \\
\hline Q3. Estacionamento & 0,782 & & & & & \\
\hline Q4. Vestiários e Banheiros & 0,694 & & & & & \\
\hline Q18. Segurança & 0,689 & & & & & \\
\hline Q10. Serviços Acessórios & 0,653 & & & & & \\
\hline Q17. Acesso & 0,613 & & & & & \\
\hline Q9. Variedade das Modalidades & 0,596 & & & & & \\
\hline Q8. Horários das Modalidades & 0,554 & & & & & \\
\hline Q11. Avaliações & 0,531 & & & & & \\
\hline Q2. Tamanho e Arquitetura dos Ambientes & 0,498 & & & & & \\
\hline Q 20. Atendimento dos Instrutores & & 0,824 & & & & \\
\hline Q 23. Interesse & & 0,798 & & & & \\
\hline Q 22. Instruções & & 0,784 & & & & \\
\hline Q 21. Qualificação & & 0,755 & & & & \\
\hline Q19. Programa de Treinamento & & 0,712 & & & & \\
\hline Q 24. Quantitativo de Funcionários & & 0,469 & & & & \\
\hline Q15. Iluminação & & & 0,736 & & & \\
\hline Q 14. Ventilação & & & 0,692 & & & \\
\hline Q13. Limpeza & & & 0,602 & & & \\
\hline Q16. Música & & & 0,595 & & & \\
\hline Q5. Variedade e Modernidade dos Equipamentos & & & 0,548 & & & \\
\hline Q6. Quantidade de Equipamentos & & & 0,521 & & & \\
\hline Q7. Arranjo físico & & & 0,477 & & & \\
\hline Q 29. Preço & & & & 0,771 & & \\
\hline Q 31. Marca & & & & 0,614 & & \\
\hline Q 26. Reclamações & & & & 0,531 & & \\
\hline Q 28. Emergência & & & & 0,512 & & \\
\hline Q1. Conservação & & & & 0,497 & & \\
\hline Q 30. Convênios, Ações de Fidelização e Planos & & & & 0,495 & & \\
\hline Q 25. Atendimento dos Func. Administrativos & & & & & 0,759 & \\
\hline Q 27. Aparência & & & & & 0,742 & \\
\hline Q.12 Localização & & & & & & 0,675 \\
\hline Percentual da variação explicada (\%) & 15,96 & 14,03 & 11,72 & 9,27 & 6,88 & 3,96 \\
\hline Percentual acumulado da variação explicada (\%) & 15,96 & 29,99 & 41,71 & 50,98 & 57,86 & 61,82 \\
\hline Alfa de Cronbach da escala (QSAG) & \multicolumn{6}{|c|}{0,940} \\
\hline
\end{tabular}

A Figura 4 apresenta a estrutura de seis dimensões que traduzem a qualidade dos serviços em academias de ginástica. 
Dimensões da Qualidade do Serviço em Academias de Ginástica

1. Estrutura física e conveniência (D1).

2. Instrutores e programa de treinamento (D2).

3. Conforto e equipamentos (D3).

4. Preço, reclamações e emergência (D4).

5. Funcionários administrativos e aparência (D5).

6. Localização (D6).

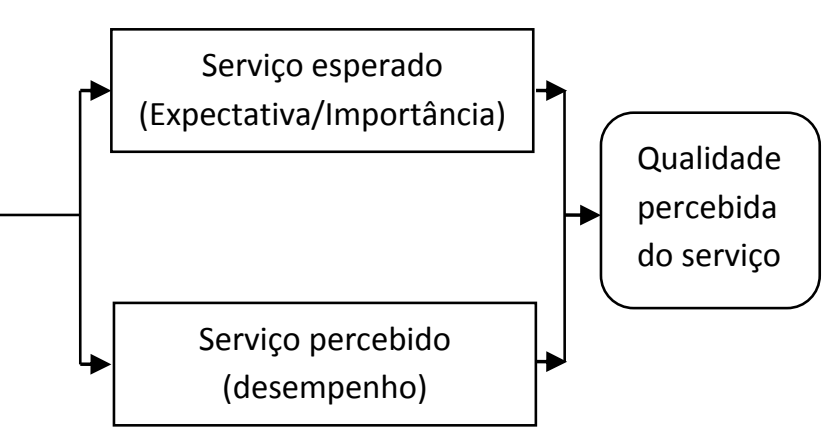

Figura 4 - Determinantes da qualidade dos serviços prestados por academias de ginástica. Fonte - Adaptado de Parasuraman, Zeithaml e Berry (1985).

\section{MATRIZ IMPORTÂNCIA-DESEMPENHO}

Considerando as dimensões da qualidade do serviço identificadas e cada zona administrativa, foram calculadas as médias nominais de desempenho $\left(M_{D}\right)$ e de importância $\left(M_{1}\right)$ para cada um dos atributos, bem como os respectivos gaps de qualidade $\left(M_{D}-M_{1}\right)$, conforme ilustra a Tabela 4.

Observou-se uma predominância de lacunas negativas, apontando que as academias estão ofertando um nível de serviço aquém do que os clientes esperam. Dos 74 gaps analisados, apenas em 13 observações (grifadas na cor cinza) o desempenho das academias sobrepujou as expectativas, com destaque para os atributos iluminação, aparência e a dimensão 5 (D5) por apresentarem apenas lacunas positivas.

Tabela 4 - Médias de desempenho e importância dos atributos por zona administrativa.

\begin{tabular}{|c|c|c|c|c|c|c|}
\hline \multirow{2}{*}{ Atributo da escala (SIGLA) } & \multicolumn{3}{|c|}{ ZS } & \multicolumn{3}{|c|}{ ZN } \\
\hline & $M_{D}$ & $M_{1}$ & GAP & $M_{D}$ & $M_{1}$ & GAP \\
\hline Dimensão 1(D1) & 8,27 & 8,63 & $-0,36$ & 7,58 & 8,28 & $-0,70$ \\
\hline Q3. Estacionamento & 7,61 & 8,71 & $-1,10$ & 6,60 & 7,99 & $-1,39$ \\
\hline Q4. Vestiários e Banheiros & 7,81 & 7,94 & $-0,13$ & 6,84 & 7,93 & $-1,09$ \\
\hline Q18. Segurança & 8,25 & 9,27 & $-1,02$ & 7,08 & 8,54 & $-1,46$ \\
\hline Q10. Serviços Acessórios & 8,49 & 7,87 & 0,62 & 7,27 & 7,51 & $-0,24$ \\
\hline Q17. Acesso & 8,77 & 8,88 & $-0,11$ & 8,33 & 8,51 & $-0,18$ \\
\hline Q9. Variedade das Modalidades & 8,54 & 8,87 & $-0,33$ & 8,37 & 8,11 & 0,26 \\
\hline Q8. Horários das Modalidades & 8,91 & 9,03 & $-0,12$ & 8,42 & 8,86 & $-0,44$ \\
\hline Q11. Avaliações & 7,80 & 8,62 & $-0,82$ & 7,38 & 8,63 & $-1,25$ \\
\hline Q2. Tamanho e Arquitetura dos Ambientes & 8,28 & 8,47 & $-0,19$ & 7,92 & 8,45 & $-0,53$ \\
\hline Dimensão 2 (D2) & 8,94 & 9,54 & $-0,60$ & 8,46 & 9,25 & $-0,79$ \\
\hline Q 20. Atendimento dos Instrutores & 9,17 & 9,68 & $-0,51$ & 8,64 & 9,43 & $-0,79$ \\
\hline Q 23. Interesse & 8,68 & 9,53 & $-0,85$ & 8,46 & 9,07 & $-0,61$ \\
\hline Q 22. Instruções & 9,11 & 9,60 & $-0,49$ & 8,65 & 9,26 & $-0,61$ \\
\hline Q 21. Qualificação & 9,16 & 9,74 & $-0,58$ & 8,69 & 9,55 & $-0,86$ \\
\hline Q19. Programa de Treinamento & 9,06 & 9,55 & $-0,49$ & 8,48 & 9,32 & $-0,84$ \\
\hline Q 24. Quantitativo de Funcionários & 8,46 & 9,12 & $-0,66$ & 7,81 & 8,85 & $-1,04$ \\
\hline Dimensão 3 (D3) & 8,28 & 8,88 & $-0,60$ & 8,20 & 8,64 & $-0,44$ \\
\hline Q15. Iluminação & 8,84 & 8,79 & 0,05 & 8,71 & 8,67 & 0,04 \\
\hline Q 14. Ventilação & 8,13 & 8,85 & $-0,72$ & 8,50 & 8,44 & 0,06 \\
\hline Q13. Limpeza & 8,97 & 9,57 & $-0,60$ & 8,61 & 9,22 & $-0,61$ \\
\hline
\end{tabular}




\begin{tabular}{|c|c|c|c|c|c|c|}
\hline Q16. Música & 7,97 & 8,41 & $-0,44$ & 8,06 & 8,43 & $-0,37$ \\
\hline Q5. Variedade e Modernidade dos Equipamentos & 7,97 & 9,12 & $-1,15$ & 7,63 & 8,57 & $-0,94$ \\
\hline Q6. Quantidade de Equipamentos & 7,80 & 9,03 & $-1,23$ & 8,00 & 8,77 & $-0,77$ \\
\hline Q7. Arranjo físico & 8,29 & 8,40 & $-0,11$ & 7,89 & 8,37 & $-0,48$ \\
\hline Dimensão 4 (D4) & 8,33 & 9,05 & $-0,72$ & 8,09 & 8,82 & $-0,73$ \\
\hline Q 29. Preço & 8,17 & 9,28 & $-1,11$ & 8,17 & 8,73 & $-0,56$ \\
\hline Q 31. Marca & 8,74 & 8,58 & 0,16 & 8,51 & 8,81 & $-0,30$ \\
\hline Q 26. Reclamações & 8,88 & 9,26 & $-0,38$ & 8,63 & 8,89 & $-0,26$ \\
\hline Q 28. Emergência & 8,17 & 9,52 & $-1,35$ & 7,74 & 9,21 & $-1,47$ \\
\hline Q1. Conservação & 8,32 & 9,19 & $-0,87$ & 8,09 & 8,77 & $-0,68$ \\
\hline Q 30. Convênios, Ações de Fidelização e Planos & 7,68 & 8,45 & $-0,77$ & 7,40 & 8,53 & $-1,13$ \\
\hline Dimensão 5 (D5) & 9,22 & 8,71 & 0,51 & 8,72 & 8,59 & 0,14 \\
\hline Q 25. Atendimento dos Funcionários Administrativos & 9,17 & 9,04 & 0,13 & 8,64 & 8,76 & $-0,12$ \\
\hline Q 27. Aparência & 9,26 & 8,38 & 0,88 & 8,80 & 8,41 & 0,39 \\
\hline Dimensão 6 (D6) & 9,39 & 9,26 & 0,13 & 8,79 & 9,09 & $-0,30$ \\
\hline Q.12 Localização & 9,39 & 9,26 & 0,13 & 8,79 & 9,09 & $-0,30$ \\
\hline
\end{tabular}

Fonte - Pesquisa (2012). Legenda: $M_{D}$ - média de desempenho; $M_{1}$ - média de importância; GAP - lacuna de qualidade; ZS - Zona Sul; ZN - Zona norte.

A matriz importância-desempenho foi empregada. O método consiste em situar os pares ordenados, formado pelos escores médios das respostas de importância e de desempenho de cada atributo, numa matriz 2 por 2 (DENG, 2008). Ao plotar as médias de importância sobre o eixo $Y$ e as médias de desempenho no eixo $X$, as quais caracterizam cada uma das dimensões extraídas, é possível identificar em quais delas ocorrem déficits de qualidade. Por meio desta ferramenta, extrai-se um conjunto de ações que podem ser usadas para dar suporte às decisões gerenciais de aperfeiçoamento das estratégias de qualidade.

Examinando a Figura 5, via de regra, as dimensões caíram em quadrantes comuns, independente da zona administrativa. As dimensões D2 e D6 situaram-se na zona adequada, mostrando que estão em níveis satisfatórios para as necessidades da empresa. Os gestores devem mantê-las, alavancá-las e promovê-las fortemente.

A dimensão D1 se posicionou na zona de melhoria, indicando que precisa ser aprimorada, porém, não como prioridade maior. A dimensão D4 caiu na zona de ação urgente, mostrando que os gestores devem tomar ações prioritárias e imediatas. Negócios provavelmente estão sendo perdidos em consequência deste fenômeno. Já a dimensão D5 situou-se na zona de excesso. Ações de marketing podem ser realizadas para induzir os clientes a valorizarem esta dimensão, ou optar em desinvestir rapidamente e remanejar os recursos.

Na dimensão D3 houve divergência em relação ao quadrante em que se posicionou, comparando as zonas administrativas. Para a zona Sul, a referida dimensão deve ser prioritariamente melhorada (zona de ação urgente), ao passo que, para a zona Norte, a mesma também necessita ser aprimorada, no entanto, não com a mesma prioridade (zona de melhoria). 


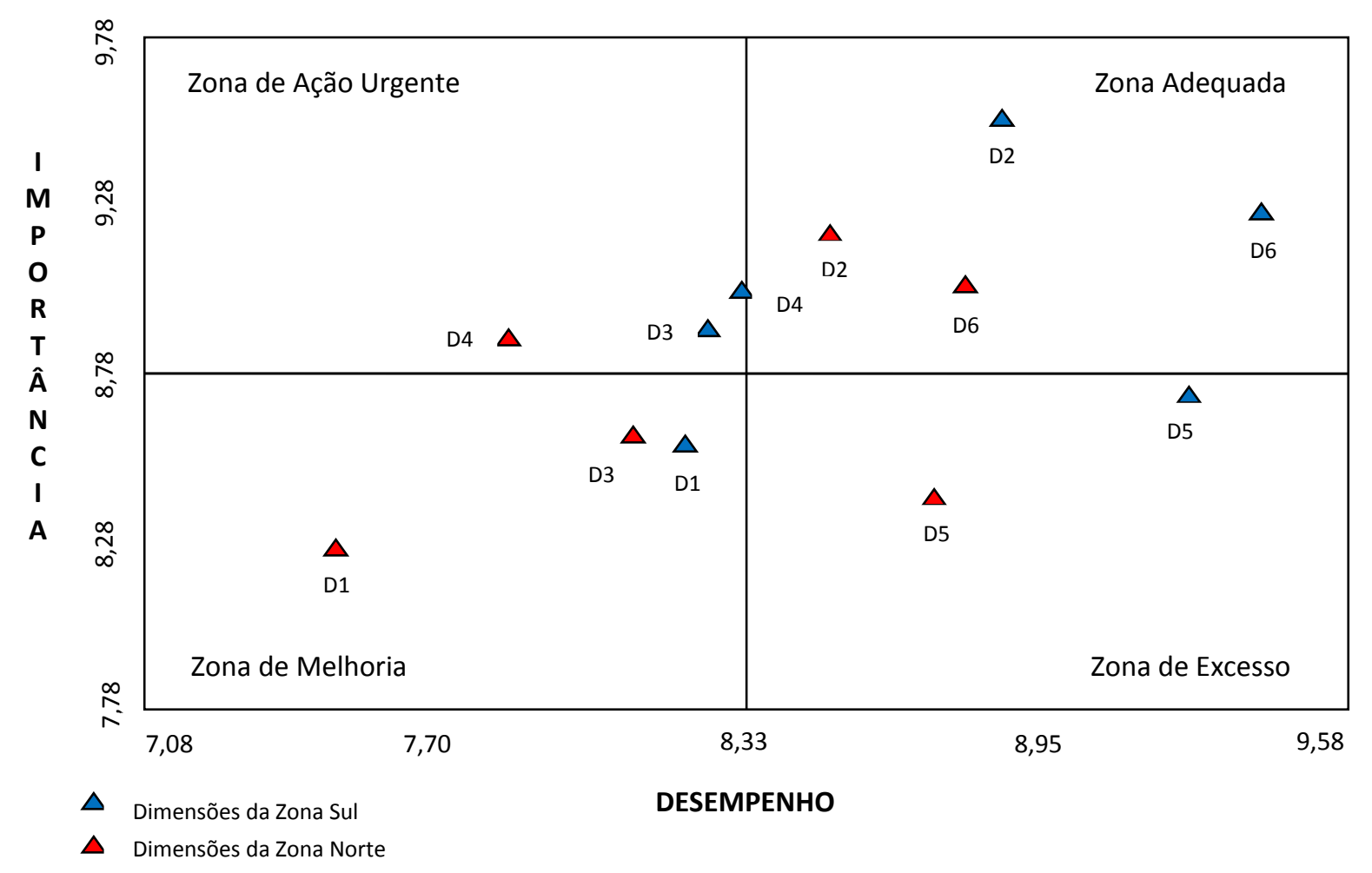

Figura 5 - Matriz importância-desempenho. Fonte - Adaptado de Yildiz (2011).

\section{CONSIDERAÇÕES FINAIS}

Este artigo visou: a) propor uma escala para mensurar a qualidade dos serviços ofertados por academias de ginástica; b) avaliar a dimensionalidade do construto qualidade do serviço e c) apontar, com base na matriz importância-desempenho, em quais dimensões os gestores devem focar sua atenção, com o propósito de aperfeiçoar o serviço ofertado. Como resultado, a escala proposta, constituída por 31 atributos e nomeada QSAG, apresentou uma consistência interna significativa (alfa de Cronbach foi 0,940). Da análise fatorial, seis fatores emergiram os quais explicam $61,82 \%$ da variância dos dados. Isto confirma a multidimensionalidade do construto qualidade dos serviços, abordada, dentre outros autores, por Parasuraman, Zeithaml e Berry (1985), Chelladurai, Scott e Haywood-Farmer (1987) e Kim e Kim (1995). Por fim, a matriz importância-desempenho permitiu apontar que os gestores das academias da zona Sul devem melhorar, prioritariamente, as dimensões D3 e D4, ao passo que, os administradores das academias da zona Norte, devem focar a sua atenção na dimensão D4. Acrescenta-se ainda que estas conclusões limitam-se às academias estudadas.

Não obstante as limitações deste estudo, o mesmo poderá contribuir para o desenvolvimento de novas investigações sobre a temática, já que foi desenvolvida uma escala para mensurar a qualidade dos serviços ofertados pelas academias de ginástica. Para tanto, sugere-se investigar, em outros recortes espaciais, a consistência da estrutura de seis fatores e o nível de confiabilidade da escala. 


\section{REFERÊNCIAS BIBLIOGRÁFICAS}

1. ACAD, Associação Brasileira de Academias. Revista ACAD Brasil, n. 56, nov. 2011. Disponível em: <www.acadbrasil.com.br>. Acesso em: 30 de maio de 2012.

2. AFTHINOS, Y.; THEODORAKIS, N. D.; NASSIS, P. Customers' expectations of service in Greek fitness centers: Gender, age, type of sport center, and motivation differences. Managing Service Quality, v. 15, n. 3, pp. 245-258, 2005.

3. AGUIAR, F. A. de. Análise da satisfação dos clientes das academias de ginástica da cidade de João Pessoa (PB). 2007. 173f. Dissertação (Mestrado em Administração) - Programa de PósGraduação em Administração, Universidade Federal da Paraíba, João Pessoa/PB, 2007.

4. AKBABA, A. Measuring service quality in the hotel industry: a study in a business hotel in Turkey. International Journal of Contemporary Hospitality Management, v. 25, p. 170-192, 2006.

5. ALEXANDRIS, K.; PALIALIA, E. Measuring customer satisfaction in fitness centres in Greece: an exploratory study. Managing Leisure, v. 4, n. 4, pp. 218-228, 1999.

6. ANDRONIKIDIS, A.; BELLOU, V. Verifying alternative measures of the service-quality construct: consistencies and contradictions. Journal of Marketing Management, v. 26, n. 5-6, pp. 570$587,2010$.

7. ASLAN, M.; KOÇAK, M. S. Determination of the service quality among sport and fitness centers of the selected universities. International Journal of Human Sciences, v. 8, n. 2, pp. 817-833, 2011.

8. BABAKUS, E.; BOLLER, G. W. An empirical assessment of the SERVQUAL scale. Journal of Business Research, v. 24, n.3, pp. 253-268, 1992.

9. BARRETO, E. G. L.; SANTOS, R. de L. S. dos; GOMES, M. de L. B.; SILVA, L. B. da. Uma análise sobre a qualidade dos serviços em uma academia de ginástica através do método SERVQUAL. In: ENCONTRO NACIONAL DE ENGENHARIA DE PRODUÇÃO, 30., 2010, São Carlos/SP, anais eletrônicos. São Carlos/SP: ENEGEP, 2010. Disponível em: <http://www.abepro.org.br/publicacoes/>. Acesso em: 11 de agosto de 2012.

10. BERTEVELLO, G. Academias de ginástica e condicionamento físico - sindicatos \& associações. 2006. Disponível em: <http://www.atlasesportebrasil.org.br/textos/146.pdf.> Acessado em: 17 de setembro de 2013.

11. BODET, G. Investigating customer satisfaction in a health club context by an application of the Tetraclasse Model. European Sport Management Quarterly, v.6, n. 2, pp. 149-165, 2006.

12. BOWERSOX, D. J.; CLOSS, D. J.; COOPER, M. B. Gestão logística de cadeias de suprimentos. Porto Alegre: Bookman, 2006.

13. BRADY, M. K.; CRONIN, J. J.; BRAND, R. R. Performace-only measurement of service quality: a replication and extension. Journal of Business Research, n. 55, pp. 17-31. 2002.

14. CHELLADURAI, P.; SCOTT, F. L.; HAYWOOD-FARMER, J. Dimensions of fitness services: development of a model. Journal of Sport Management, v. 1, n.1, pp. 159-172, 1987.

15. COLMENARES, O. A.; SAAVEDRA, J. L. Aproximación teórica de los modelos conceptuales de la calidad del servicio. Técnica Administrativa, v. 6, n. 4, 2007. 
16. CONSELHO REGIONAL DE EDUCAÇÃO FÍSICA - CREF 10 REGIÃO. Pessoas jurídicas registradas. Disponível em: <http://www.cref10.org.br>. Acesso em: 26 de julho de 2012.

17. CRONIN, J. J.; TAYLOR, S. A. Measuring service quality: a reexamination and extension. Journal of Marketing, v. 56, July, pp. 55-68. 1992.

18. DENG, Wei-Jaw. Fuzzy importance-performance analysis for determining critical service attributes. International Journal of Service Industry Management, v. 19, n. 2, pp. 252-270, 2008.

19. DIAS, D.M. F. Influência da qualidade/satisfação na academia de fitness de um corpo de bombeiros e suas implicações. 2006. 222 f. Dissertação (Mestrado em Ciências do Desporto) Universidade do Porto, Porto, Portugal, 2006.

20. FURTADO, R. F. Do fitness ao wellness: os três estágios de desenvolvimento das academias de ginástica. Pensar a Prática, v. 12, n. 1, pp. 1-11, 2009.

21. GRÖNROOS, C. A service quality model and its marketing implications. European Journal of Marketing, v. 18, n. 4, pp. 36-44, 1984.

22. GRÖNROOS, C. Marketing: gerenciamento e serviços. 3. ed. Rio de Janeiro: Elsevier, 2009.

23. HAIR JUNIOR, J.F.; BLACK, W.C.; ANDERSON, R.E.; TATHAM, R.L. Análise multivariada de dados. Porto Alegre: Bookman, 2009.

24. INTERNATIONAL HEALTH, RACQUET \& SPORTSCLUB ASSOCIATION (IHRSA). The 2012 IHRSA global report analyzes industry performance and outlook. EUA, maio 2012. Disponível em: <www.ihrsa.org>. Acesso em: 30 de maio de 2012.

25. JAIN, S. K.; GUPTA, G. Measuring service quality: SERVQUAL vs. SERVPERF scales. Vikalpa, v. 29, n. 2, April - June, pp. 25-37, 2004.

26. KIM, D.; KIM, S.Y. QUESC: an instrument for assessing the service quality of sport centers in Korea. Journal of Sport Management, v. 9, n. 2, pp. 208-220, 1995.

27. LAGROSEN, S.; LAGROSEN, Y. Exploring service quality in the health and fitness industry. Managing Service Quality, v. 17, n.1, pp. 41-53, 2007.

28. LAM, E. T. C.; ZHANG, J. J.; JENSEN, B. E. Service quality assessment scale (SQAS): an instrument for evaluating service quality of health-fitness clubs. Measurement in Physical Education And Exercise Science, v. 9, n. 2, pp. 79-111, 2005.

29. MORENO, F. C.; GARCÍA, N. M.; POMAR, J. N. Una aplicación inicial del modelo tridimensional de calidad de servicio en centros deportivos privados. Revista de Ciencias del Deporte, v. 8, n. 1, pp. 67-81, 2012.

30. MURRAY, D.; HOWAT, G. The relationships among service quality, value, satisfaction, and future intentions of customers at an Australian sports and leisure centre. Sport Management Review, v. 5, n. 1, pp. 25-43, 2002.

31. OSTI, L. R. de. Atributos de satisfação como determinantes da lealdade de clientes de academias no Brasil. 2009. 93f. Dissertação (Mestre em Gestão do Desporto) - Programa de Pós-Graduação em Gestão do Desporto, Universidade Técnica de Lisboa, Portugal, 2009. 
32. PAPADIMITRIOU, D. A.; KARTEROLIOTIS, K. The service quality expectations in private sport and fitness centers: a reexamination of the factor structure. Sport Marketing Quarterly, v. 9, n. 3, pp. 157-164, 2000.

33. PARASURAMAN, A; ZEITHAML, V. A.; BERRY, L. L. A conceptual model of service quality and its implications for future research. Journal of Marketing, v. 49, pp. 41-50, 1985.

34. PARASURAMAN, A.; ZEITHAML, V. A.; BERRY, L. L. Reassessment of expectations as a comparison standard in measuring service quality: implications for further research. Journal of Marketing, v. 58, n.1, p. 111-124, 1994.

35. PEDRAGOSA, V.; CORREIA, A. Expectations, satisfaction and loyalty in health and fitness clubs. International Journal of Sport Management and Marketing, v. 5, n. 4, pp.450-464, 2009.

36. PINHEIRO, I. A; PINHEIRO, R. R. Organização científica do trabalho reinventa um mercado tradicional: o caso do fitness. RAE-eletrônica, v. 5, n. 2, 2006

37. SABA, F. Liderança e gestão: para academia e clubes esportivos. São Paulo: Phorte, 2006.

38. SOITA, P. W. Customers' perception about service quality in commercial health and fitness clubs in Uganda. Journal of Education and Practice, v. 3, n. 4, pp. 53-63, 2012.

39. TEIXEIRA, M.; CORREIA, A. Segmenting fitness centre clients. International Journal of Sport Management and Marketing, v. 5, n. 4, pp. 396-416, 2009.

40. THEODORAKIS, N.; ALEXANDRIS, K.; RODRIGUEZ, P.; SARMENTO, P. J. Measuring customer satisfaction in the context of health clubs in Portugal. International Sports Journal, n 8, v. 1, pp. 44-53, 2004.

41. TRIADO, X. M.; APARICIO, P.; RIMBAU, E. Identification of factors of customers' satisfaction in municipal sport centres in Barcelona: some suggestions for satisfaction improvement. Cyber Journal of Sport Marketing, v. 3, n. 4, 1999.

42. UHLMANN, V. C.; PETRI, A. A. M.; DAMKE, L.; DILLMANN, C.; ZAMBERLAN, L. Motivações e a satisfação de usuários de academias de ginástica. In: SemeAd - Seminário em Administração, 12., 2009, São Paulo, anais eletrônicos. São Paulo: SemeAd, 2009. Disponível em: <www.ead.fea.usp.br>. Acesso em: 27 de janeiro de 2012.

43. YILDIZ, S. M. An importance-performance analysis of fitness center service quality: empirical results from fitness centers in Turkey. African Journal of Business Management , v. 5, n. 16, pp. 7031-7041, 2011.

44. YILDIZ, S. M; KARA, A. A re-examination and extension of measuring perceived service quality in physical activity and sports centres (PSC): QSport-14 scale. International Journal of Sports Marketing \& Sponsorship, v. 13, n. 3, pp. 189-208, 2012. 